\title{
A Survey on Physical MIMo Channels with tranceiver imperfections
}

\author{
P.V.S Muralidhar, T.D Prashanthi \\ M.Tech Student, Assistant Professor
}

\begin{abstract}
The capability of perfect MIMO channels has a high SNR grade that equals the minimum of the add up to of transmit and receive stsam. This is due to the fact that, unlike base stations, relays are low-cost nodes that can be easily deployed and, hence, enhances the network agility. The vast majority of works in the context of relaying networks make the assumption of ideal transceiver hardware. The vast majority of technical contributions in the area of relaying assume ideal transceiver hardware. Technological advances can reduce transceiver impairments, but there is currently an opposite trend towards small low-cost low-power transceivers where the inherent dirty RF effects are inevitable and the transmission is instead adapted to them. We prove analytically that such physical MIMO channels have a finite upper capacity limit, for any channel distribution and SNR. The high-SNR slope thus collapses to zero. One explanation is the finite channel coherence time that limits the resources for channel acquisition and coordination between nodes, thus creating a finite fundamental ceiling for the network spectral efficiency-irrespectively of the power and the number of antennas.
\end{abstract}

sannetna , snoitats esab , erawdrah , RNS

\section{Introduction}

Wireless communication enjoys considerable attention in the research community. Recent advances are mainly market driven by the demand for applications with increased data rates. Especially, wireless local area networks (WLANs), which aim at replacing wired computer network infrastructure with wireless communication technology, seem to raise a strong demand for further research and development.

Different approaches to boost WLAN data rates have been considered in the past, as reflected in the amendments of the Institute of Electrical and Electronics Engineers (IEEE) 802.11 standards, First, data rates up to $11 \mathrm{Mbit} / \mathrm{s}$ are supported by IEEE $802.11 \mathrm{~b}$ compliant equipment. The modulation is direct sequence spread spectrum-based, which renders wireless channel equalization a complex task in the receiver. Unlike the conventional point-to-point channels, in a wireless network, the overall throughput of the system is interference limited.

That is, boosting up the transmitted power of a user cannot efficiently increase the spectrum efficiency of the network, since strong signals transmitted by one user acts as strong interference on other users. Therefore, it is of interest to develop approaches to increase the spectrum efficiency without increasing the transmitted power. With the introduction of orthogonal frequency-division multiplexing (OFDM) [15] techniques in the popular IEEE 802.11a and IEEE 802.11 g standards, data rates up to $54 \mathrm{Mbits} / \mathrm{s}$ in a bandwidth of $20 \mathrm{MHz}$ can be realized with low complexity channel equalization. Channel bonding, i.e., expanding the bandwidth from 20 $\mathrm{MHz}$ to $40 \mathrm{MHz}$, doubles the data throughput in some systems.

OFDM has been employed in other standards as well due to its suitability for transmission over wireless links that exhibit frequency selectivity. These include standards for metropolitan area networks such as the IEEE 802.16 (WiMAX) standard. Even for broadcast systems, OFDM is becoming increasingly important. This manifests itself through the introduction of digital radio mondiale and digital audio broadcast in the shortwave bands and high-frequency bands, respectively. For video broadcasting, OFDM with advanced data compression techniques is also set to replace legacy analog transmission schemes.

While higher throughput is anticipated, the available bandwidth for wireless systems is generally limited. This calls for technologies that achieve a higher throughput per bandwidth, i.e., higher spectral efficiency. Especially in crowded places, such as airports, train stations, or convention centers, low system capacity provided by today's technology poses a problem, which is the cause for insufficient data rates to individual users demanding basic data services.

\section{Mimo Technology}

MIMO technology allows multiple antennas at both transmitter and receiver to transmit independent data streams concurrently in the same frequency band (see Figure 1). This principle, generally known as spatial multiplexing, results in a significantly higher spectral efficiency compared to single-input single-output (SISO) systems due to spatial diversity enabling a multiplexing gain. However, the detection algorithms employed in 
MIMO receivers have generally higher computational and hardware complexity than traditional SISO detection algorithms as demonstrated.

MIMO technology is about to gain access to the consumer market through its introduction in several standards. These include:

- 802.11n: MIMO extension for the 802.11a WLAN standard that supports spatial multiplexing of up to four streams and data rates of up to $600 \mathrm{Mbit} / \mathrm{s}$.

- 802.16e (WiMAX): fixed broadband wireless access.

- UMTS: MIMO add-on for the existing 3G mobile standard. This constitutes an evolutionary step toward 4G networks.

- 802.2: future standard that shall include MIMO that meets the requirements for high mobility outdoor scenarios in an allIP network in licensed frequency bands.

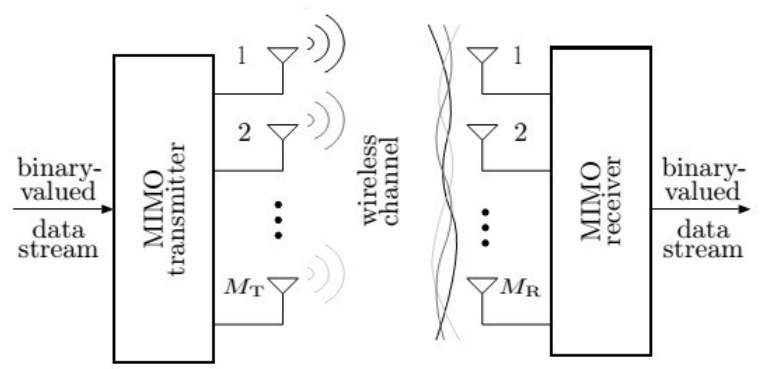

Fig 1 Illustration of a MIMO system model withMRreceive and MTtransmit antennas.

The capacity of ideal MIMO channels has a high SNR slope that equals the minimum of the number of transmit and receive antennas. This letter analyzes if this result holds when there are distortions from physical transceiver impairments. We prove analytically that such physical MIMO channels have a finite upper capacity limit, for any channel distribution and SNR. The high-SNR slope thus collapses to zero. This appears discouraging, but we prove the encouraging result that the relative capacity gain of employing MIMO is at least as large as with ideal transceivers.

This work analyzes the generalized MIMO channel with transceiver impairments. We show that the capacity has a finite high-SNR limit for any channel distribution. The multiplexing gain is thus zero, which is fundamentally different from the ideal case in (detailed above). Similar single antenna results are give. The practical MIMO gain - the relative capacity increase over single-antenna channels - is however shown to be at least as large as with ideal transceivers.

The parameters to be estimated and subsequently compensated or used to enhance detection include:

- Carrier frequency and sampling rate offset: caused by reference oscillator frequency differences between transmitter and receiver.

- Frame timing: in frame-based communication systems the start of a data packet transmission must be synchronized.

- Signal-to-noise ratio (SNR), signal-to-interference-plus-noise ratio (SINR): these parameters are used in estimation algorithms that employ for example maximum ratio combining (MRC).

- Received signal power: due to a fading channel the dynamic range of the received signal power is large.

- MIMO channel coefficients: needed to allow spatial separation of the received signals.

- Common phase noise: caused by jitter in the reference oscillators and phase locked loops (PLL) at the receiver and transmitter.

Generally, hardware resources for parameter estimation should be minimized since dedicated estimation circuitry is idle during most of the time. Here, digital signal processor (DSP) based approaches pose an advantage as the same hardware can be used for all tasks during frame reception. his calls for an evaluation of available estimation algorithms for efficient implementation. However, the hardware resources including radio frequency (RF) equipment have to be considered as well, as imposed constraints influence the algorithms considerably. Finally, the ensemble of various estimation and compensation procedures has to provide satisfactory results, as algorithms may interact and reveal adverse effects when running simultaneously.

\section{System Model}

This chapter deals with the theory and principles used throughout this thesis. First, the mathematical notation is introduced followed by the fundamentals of OFDM. These concepts are applied to a frame-based MIMO-OFDM system model considering all signal processing stages from mapping bits to constellations in the transmitter to the estimation of these constellations in the receiver. Only basic impairments, such as a quasistatic frequency-selective channel are considered in this model. Subsequently, real-world impairments are 
introduced that dominate indoor frame-based systems with rather large tone-spacing such as OFDM-based WLAN systems described in the IEEE 802.11 standards.

\subsection{Notation}

This section outlines the notation used in this thesis. An exhaustive list of all operators and variables is given in Appendix A. A matrix is denoted by a bold uppercase letter. The entry of matrix A in the $r_{\text {th }}$ row and $c_{\text {th }}$ column is denoted by $a_{r}, c$. The $c_{t h}$ column of matrix $A$ is denoted by $a_{c}$. Lowercase bold letters denote vectors. Therth entry of vector a is denoted by $\mathrm{a}_{\mathrm{r}}$.

To describe MIMO-OFDM systems in vector notation, three dimensions are required. This can be introduced making use of brackets[']. Therefore, $\mathrm{A}[\mathrm{m}]$ denotes the $\mathrm{m}_{\mathrm{th}}$ matrix of a collection of $\mathrm{M}$

matrices. The $c, r_{\text {th }}$ element of the $m_{\text {th }}$ matrix is denoted by $a_{r}, c[m]$. This extra dimension describes usually the time or frequency index. $a_{\mathrm{r}}, c[\cdot]$ denotes $\mathrm{aM} \times 1$ column vector containing all entries $a_{\mathrm{r}}, c[\mathrm{~m}]$ wherem $=0,1, \ldots, \mathrm{M}-1$. See Figure 2.1 for an illustration of the notation.

$$
p(x)=\frac{1}{\sqrt{2 \pi \sigma^{2}}} \exp \left[\frac{(x-\mu)^{2}}{2 \sigma^{2}}\right] .
$$

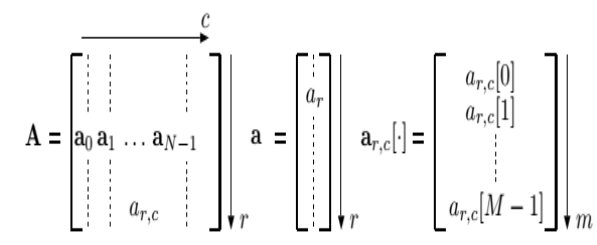

Fig 2 Notation of matrices (left) and vectors (middle). To obtain vectors from entries of a collection of matrices the notationar, $\mathrm{c}[\cdot]$ is used (right).

Brackets may also be used to access individual entries of a vector; thus, the $r_{\text {th }}$ entry of a vector a is denoted as $\mathrm{a}[\mathrm{r}]=\mathrm{ar}$. Occasionally, the index exceeds the defined length of a vector. We assume all vectors to be virtually expanded with zeros, thus returning( $)$ in these cases. The symbols $\mathrm{w}$ and $\mathrm{k}$ denote indices in the frequency domain and time domain, respectively. Constants, operators, and identifiers of variables are always written in upright font, for example, $\mathrm{A}_{\mathrm{hs}}$ denotes the Hermitian transposition of a matrix A with label s.

Gaussian Distribution: A very important distribution encountered frequently in communication systems is the Gaussian distribution. This distribution is denoted by $\mathrm{N}\left(\mu, \sigma^{2}\right)$, where $\mu$ corresponds to the mean and $\sigma^{2}$ to the variance. A random variable $X \sim N\left(\mu, \sigma^{2}\right)$ is Gaussian distributed. Its probability density function is given by

Discrete Fourier Transform: OFDM modulation is performed using the discrete Fourier transformation (DFT). The unitary and therefore power-neutral DFT of a vectorf $=$ DFT $(t)$ is defined as

$$
\mathrm{f}=\mathrm{Wt} \quad(2)
$$

where the $\mathrm{N} \times \mathrm{N}$ matrix $\mathrm{W}$ defines the unitary discrete Fourier transform

$$
w_{r, c}=\frac{1}{\sqrt{N}} \exp \left(-\mathrm{j} 2 \pi \frac{r c}{N}\right) .
$$

If $\mathrm{N}$ is chosen as a power of two, the much more efficient fast Fourier transform (FFT) is used instead of the DFT in order to lower the computational complexity. The inverse unitary discrete Fourier transformation is given by

$$
\mathrm{t}=\mathrm{W}^{\mathrm{H}} \mathrm{f} \text {. (4) () }
$$

\subsection{Baseband MIMO-OFDM Model}

In this section, a frame-based MIMO-OFDM system model is introduced. The structure of this MIMO Model is organized in order to be compatible with the IEEE 802.11 system description. Spatialmultiplexing is employed, where multiple, independent streams are transmitted in parallel in the same frequency band at the same time. The data is transmitted in frames. This allows multiple users to access the same physical resources easily by means of time division multiple access (TDMA). The MIMO system under consideration employs MT antennas at the transmit terminal andMRantennas at the receive terminal as outlined in Figure 2.2 and Figure 2.1 , respectively. For a spatial multiplexing system to function efficiently, we impose that the number of receive antennasMRalways exceeds or equals the number of transmit antennas MT. 


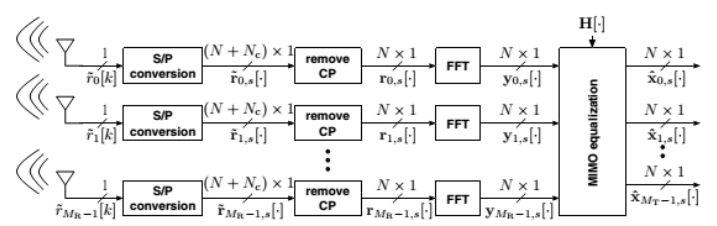

Fig 3 MIMO-OFDM receiver with MR parallel SISO-OFDM receivers. MIMO equalization involves all MR streams concurrently and yields estimates of the M Ttransmitted streams.

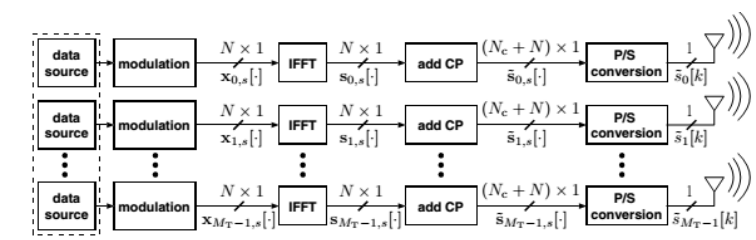

Fig 4 MIMO-OFDM transmitter based on MTparallel SISOOFDM transmitters. Note that the data sources may have dependencies if coding over the spatial dimension is used.

The signals involved in the MIMO Model are band limited. This allows describing the signals in a discretized form. To transform signals from the time domain into the frequency domain, the discrete Fourier transform can be applied. If the sampling rate is $\mathrm{f}_{\mathrm{s}}$, the Nyquist theorem states that the maximal reconstructible frequency is $\mathrm{f}_{\mathrm{s}} / 2$. However, with a complex-valued signal, the negative frequencies may also be modulated independently, expanding the reconstructible frequency range by a factor of 2 starting fromT ${ }^{-1} \mathrm{fs} / 2 \mathrm{tofs} / 2$. This allows to describe an N-tone OFDM symbol by $\mathrm{N}$ samples in both the time and the frequency domain. The maximum system bandwidth $\mathrm{B}$ is defined by the sampling rate $\mathrm{B}=\mathrm{f}_{\mathrm{s}}$. The number of tones $\mathrm{N}$ used by the system defines the period of a plain OFDM symbol to $\mathrm{T}=\mathrm{N} / \mathrm{f}_{\mathrm{s}}$ as well as the tone spacing $\mathrm{T}^{-1}=\mathrm{f}_{\mathrm{s}} / \mathrm{N}$. In the case of IEEE 802.11a, tones on the edge of the used communication band are assigned zero energy to ease a separation of adjacent channels.

In the transmitter, multiple OFDM-symbols are modulated to form a frame for transmission. The MT transmit signals are superimposed in the effective channel and picked up by the receive antennas. The receiver first removes the cyclic prefix and transforms the remaining signal into the frequency-domain using an FFT. A MIMO equalizer finally estimates the transmitted data stream on each tone independently.

\subsubsection{MIMO Transmitter}

The transmitter can be partitioned into multiple parallel SISO-OFDM transmitters (see Figure 2.2). An OFDM-frame is comprised of many OFDM symbols that serve different purposes in the receiver. Each OFDM symbol with $\mathrm{N}$ available tones is first modulated in the frequency domain. The binary data bits are mapped to complex-valued symbols for each non-zero tone. The set of allowed symbols depends on the modulation mode employed. In the case of binary phase shift keying (BPSK), only one bit is transmitted on each tone, while QPSK, 16-QAM, and 64-QAM allow to transmit 2 bits, 4 bits, and 6 bits per tone, respectively (see Figure 2.3). The constellation sets are chosen such that the average power on each used tone is one. The sets are denoted by $\mathrm{O}_{\mathrm{o}}$, whereo $\in\{0,1,2,4,6\}$ defines the number of bits used to identify one constellation set. $\mathrm{N}$ tones form the OFDM symbol $\mathrm{a}_{\mathrm{T}}, \mathrm{s}[\cdot]$ on transmit antennaa $\mathrm{T}$ in the frequency domain. Thus, each complex-valued entry in the vector xaT,s $[\cdot]$ defines the amplitude and phase of an oscillation at the corresponding frequency during the $\mathrm{s}_{\mathrm{th}}$ symbol on transmit antennaa $\mathrm{T}=0,1, \ldots, \mathrm{MT}-1$.
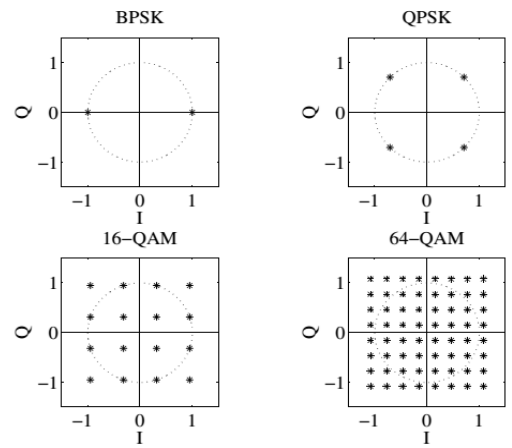

Fig 5 Sets of complex constellations used for modulation: O1(top left), O2(top right), O4(bottom left), and O6(bottom right). The unity circle is drawn for orientation in each plot. 


\subsubsection{Effective MIMO Channel}

Many tasks performed by the MIMO-OFDM receiver are similar to a SISO-OFDM receiver (see Figure 2.1): the OFDM symbols are extracted from the MR received signal sample streams based on a genie aided reference sample numberk0 (i.e., for the time being perfect frame-timing is assumed in order to avoid the discussion of non relevant cases of frame-timing). This is followed by the cyclic prefix removal and subsequent FFT calculation. After that, MIMO equalization, which is far more complex as in the processing required in the SISO case, poses the largest difference to a SISO-OFDM receiver.

\section{System Impairments}

This thesis focuses on algorithms to estimate and compensate impairments in real-world MIMOOFDM communication-system implementations. However, different impairments have different impacts on a given communication system throughput. While some impairments dominate in terms of SINR loss, others are not limiting at all. In order to understand the effects of the impairments that have to be considered in a frame based MIMO system, models are required that consider only those impairments that ultimately limit the system's performance. This allows focusing on impairments that pose a real bottleneck in a given system. In the following, impairments are considered, which occur in indoor OFDM systems with rather large tone-spacing, as it is the case for example with IEEE 802.11 based WLAN systems. The effects of these impairments are included in the MIMO Model. Then, the respective effects on the received signal ${ }^{\wedge} \mathrm{X}[\cdot]$ are characterized.

The impairments and effects identified to be important in this system model are: effective MIMO channels, thermal noise, carrier frequency offset, sampling rate offset, and phase noise. An overview of all considered impairments is given in Figure 2.4. IQ imbalance, which is a common impairment incurred by analog IQ demodulators, is not considered, since it is completely avoided in our system making use of a digital IQ de/modulator.

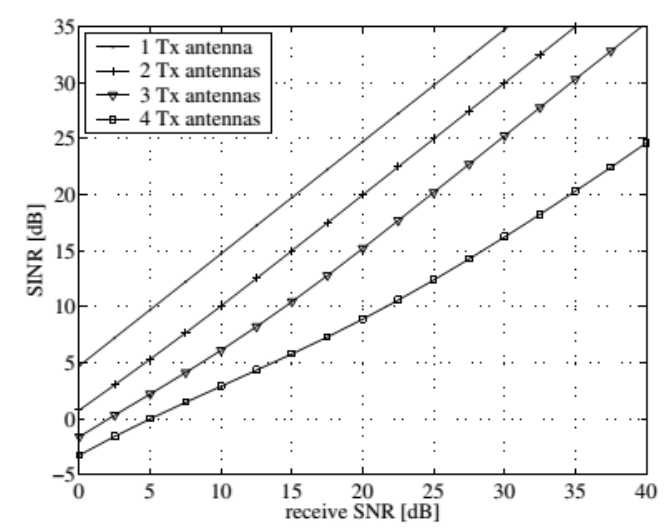

Fig 6 Expected SINR levels for a perfectN×4MIMO-OFDM system with MMSE estimators for MIMO equalization and Rayleigh fading channels.

The impairments and effects identified to be important in this system model are: effective MIMO channels, thermal noise, carrier frequency offset, sampling rate offset, and phase noise. An overview of all considered impairments is given in Figure 2.4. IQ imbalance, which is a common impairment incurred by analog IQ demodulators, is not considered, since it is completely avoided in our system making use of a digital IQ de/modulator.

\section{Channel Capacity}

In a fixed wireless environment, the fading coefficients vary slowly, so the transmitter can periodically send pilot signals to allow the receiver to estimate the coefficients accurately. In mobile environments, however, the fading coefficients can change quite rapidly and the estimation of channel parameters becomes difficult, particularly in a system with a large number of antenna elements. In this case, there may not be enough time to estimate the parameters accurately enough. Also, the time one spends in sending pilot signals is not negligible, and the tradeoff between sending more pilot signals to estimate the channel more accurately and using more time to communicate to get more data through becomes an important factor affecting performance. In such situations, one may also be interested in exploring schemes that do not need explicit estimates of the fading coefficients. It is therefore of interest to understand the fundamental limits of non-coherent multiple antenna communications. 


$$
\mathbf{Y}=\sqrt{\frac{\mathrm{SNR}}{M}} \mathbf{H X}+\mathbf{W}
$$

with the only difference that $\mathrm{H}$ is not known at the receiver, as well as the transmitter. The channel capacity and error probability are defined similarly to their coherent counterparts. The computation is, however, usually much more complicated. Some partial results are

\subsection{Channel Capacity}

The Shannon capacity of a time-invariant channel is defined as the maximum mutual information between the channel input and output. This is the maximum data rate that can be transmitted over the channel with arbitrarily small error probability. When the CSI is perfectly known at both the transmitter and the receiver, the transmitter can adapt its transmission strategy relative to the instantaneous channel state. If the channel is time variant, the ergodic capacity is the maximum mutual information averaged over all channel states. The ergodic capacity is typically achieved using an adaptive transmission policy where the power and data rate vary relative to the channel state variations. In a multiple user scenario, MU MIMO allows the reuse of time and frequency resources. Due to the scattering in different scenarios, the users' wavefronts may have large angle spreads and random signatures. Therefore, even users that are well separated in angle may have potentially overlapping subspaces spanned by left singular vectors of their channel matrices. Separability of their subspaces is much more difficult to achieve.

In a single-user MIMO system the link is point-to-point with a defined capacity. In a multi-user MIMO system, the link is a multiple access channel on the uplink and broadcast channel on the downlink. The achievable rates are characterized in this case in terms of a sum rate region. SU MIMO suffers only a small penalty in information rate without CSI at the transmitter. MU MIMO has a much larger penalty on the downlink. In a SU MIMO system, precoding at the transmitter and decoding at the receiver can be done with full cooperation between the collocated antennas. In a MU MIMO system, the antennas can cooperate at the base station for precoding on the downlink and for decoding on the uplink. However, the users cannot cooperate in decoding on the downlink or during the precoding on the uplink. In a MU MIMO system, cooperation between the users may be possible in terms of power rates assigned to the users. In a SU MIMO system, the information rate is identical on the uplink and downlink for the same transmit power if the channel is known at the transmitter and the receiver.

\subsection{Single-user MIMO capacity}

When the channel is constant and known perfectly at the transmitter and the receiver, the capacity of the system defined by

$$
C_{S U}=\max _{\boldsymbol{F}: \operatorname{Tr}\left(\boldsymbol{F} \boldsymbol{R}_{x} \boldsymbol{F}^{H}\right) \leq P_{T}} \log \frac{\operatorname{det}\left(\boldsymbol{R}_{n}+\boldsymbol{H} \boldsymbol{F} \boldsymbol{R}_{\boldsymbol{x}} \boldsymbol{F}^{H} \boldsymbol{H}^{H}\right)}{\operatorname{det}\left(\boldsymbol{R}_{n}\right)}
$$

where $R_{x}$ and $R_{n}$ are the input data correlation matrix and the noise correlation matrix. PT is the maximum transmit power. It was shown, that the optimum strategy to achieve maximum information rate is to convert the MIMO channel to parallel, non-interfering SISO channels through a singular value decomposition (SVD) of the channel matrix. The SVD yields min (MR, MT ) parallel channels with gains corresponding to the singular values of $\mathrm{H}$.

The union of achievable rates under all transmission strategies is called the capacity region of the multi-user system. It defines the limit of error-free communications given certain channel characteristics and it is used as the ultimate measure of channel capacity.

The optimal iterative water pouring algorithm was first proposed. At each step of the algorithm, one user optimizes his precoding matrix while treating the signals from all other users as interference. In the next step, the next user in numerical order optimizes his precoding matrix while treating all other users, including the updated precoding matrix of the previous user, as interference. The optimal ordering of users is independent of the channel state.

This is Sato's upper bound on the capacity region of general broadcast channels, which is the capacity of a system where the users in the downlink can cooperate. The Sato bound is not tight in general, but by introducing noise correlation at the different receivers, we can get a much stronger bound. The downlink problem at the BS is to broadcast the user signals with appropriate processing and spatial weighting, such that each user receives a maximum or desired signal-to-interference and noise ratio (SINR), information rate or BER. Antennas at the base station can cooperate during the encoding phase. Cooperation between the users might either entail cooperative management of the rates or SINR at each user. The capacity region of the general non-degraded broadcast channels is unknown. However, in it was shown that Costa's "dirty-paper" coding is optimal in achieving the sum-rate capacity, by demonstrating that the achievable rate meets the Sato upper 
bound. The basic premise of DPCs is that if the transmitter has perfect, non-causal knowledge of additive Gaussian interference in the channel, then the capacity of the channel is the same as if there was no additive interference. DPC allow non-causally interference to be"pre-subtracted" at the transmitter, but in such a way that the transmit power is not increased.

It is easily seen that the objective function for the DPC sum-rate capacity region is not a convex function of the correlation matrices. Thus, numerically finding the maximum is not a trivial problem and requires a brute force search over the entire space of correlation matrices that meet the power constraint. However, by establishing the duality between the uplink and the downlink, it was shown that it is possible to obtain the maximum achievable sum-rate capacity of the broadcast channel from the dual uplink channel. The channel capacity is different for the uplink and the downlink due to the fundamental differences between these channels. However, the fact that the downlink and the uplink channels look like mirror images of each other implies that there is a duality between these channels that allows the capacity region of either channel to be obtained from the capacity region of the other.

The equivalence between the performance of receive and transmit strategies when the roles of transmitters and receivers are reversed for vector Gaussian channels has been observed in many different situations. In a point-to-point communication, the capacity is unchanged when the role of transmitters and receivers is interchanged. In case of downlink linear processing followed by SU receivers at the UTs, the choice of transmit and receive matrices is closely related to a virtual uplink problem.

Theorem 1. The asymptotic capacity limit $\mathrm{CNt}, \mathrm{Nr}(\infty)=\lim \mathrm{SNR} \rightarrow \infty \mathrm{CNt}, \mathrm{Nr}(\mathrm{SNR})$ is finite and bounded as

$$
M \log _{2}\left(1+\frac{1}{\kappa^{2}}\right) \leq C_{N_{t}, N_{r}}(\infty) \leq M \log _{2}\left(1+\frac{N_{t}}{M \kappa^{2}}\right)
$$

This theorem shows that physical MIMO systems have a finite capacity limit in the high-SNR regime - this is fundamentally different from the unbounded asymptotic capacity for ideal transceivers. Furthermore, the bounds in hold for any channel distribution and are only characterized by the number of antennas and the level of impairments $\kappa$.

These channels perform similarly and have the same capacity limit when $\mathrm{Nt}=4$. The convergence to the capacity limit becomes faster for the random distribution when Nt increases, but the value of the limit is unchanged. Contrary, the capacity limits in the deterministic cases increase with $\mathrm{Nt}$ (and with $\alpha$ since it makes the distortion more isotropic). Fig. 3 shows that there is a medium SNR range where the capacity exhibits roughly the same M -slope as achieved asymptotically for ideal transceivers. Following the terminology of [3], this is the degrees-of-freedom (DoF) regime while the high-SNR regime is the saturation regime; see Fig. 3. This behavior appeared in for large cellular networks due to limited coherence time, but we demonstrate its existence for any physical MIMO channel (regardless of size) due to transceiver impairments.

\section{Tx-Rx Impairments}

MIMO wireless communication systems have attracted considerable attention over the past decades due to their ability to enhance the channel capacity and transmission reliability. Telatar and Foschini have respectively and that there is a linear growth in channel capacity by increasing the number of transmit and receive antennas, without requiring additional transmit power or bandwidth. Although numerous publications have appeared in this field, the vast majority assumes ideal RF hardware.

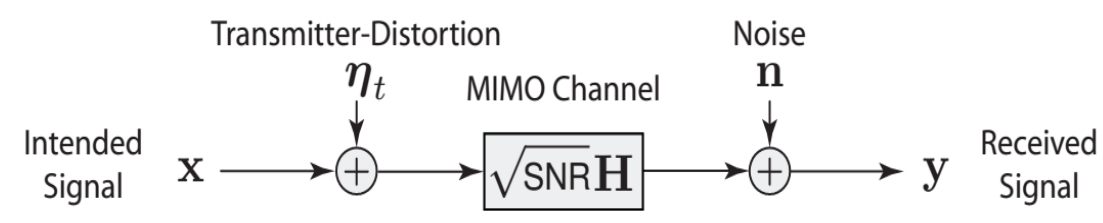

Fig 7.: Block diagram of the generalized MIMO channel considered in this letter. Unlike the classical channel model, the transmitter distortion generated by physical transceiver implementations is included in the model.

Physical radio-frequency (RF) transceivers suffer from amplifier non-linearities, IQ-imbalance, phase noise, quantization noise, carrier-frequency and sampling-rate jitter/offsets, etc. These impairments are conventionally overlooked in information theoretic studies, but this letter shows that they have a non-negligible and fundamental impact on the spectral efficiency in modern deployments with high SNR. This letter analyzes the generalized MIMO channel with transceiver impairments from [7]. We show that the capacity has a finite high-SNR limit for any channel distribution. The multiplexing gain is thus zero, which is fundamentally different from the ideal case in [1] (detailed above). Similar single antenna results are given in [5]. The practical MIMO gain - the relative capacity increase over single-antenna channels - is however shown to be at least as large as with ideal transceivers. 
However, this assumption is quite unrealistic in practice. More specifically, RF impairments, such as I/Q imbalance HPA nonlinearities and oscillator PN are known to have a deleterious impact on the performance of practical MIMO systems. Even though one can resort to calibration schemes at the transmitter, or compensation algorithms at the receiver to partially mitigate these impairments [9], there still remains certain amount of distortion unaccounted for. The reasons for such residual transceiver impairments are, for example, inaccurate models which are used to characterize the impairments' behavior, imperfect parameters estimation errors due to thermal noise, and unsophisticated compensation algorithms with limited capabilities.

In this context, very few publications have studied the impact of residual transceiver impairments. For example provided experimental results to model the statistical behavior of residual hardware impairments. Moreover, they also investigated the impact of transmitter impairments on several existing MIMO detection algorithms (e.g., zero-forcing detection, maximum-likelihood detection, and max-log a posteriori probability detection)

The authors analyzed the MIMO channel capacity under the aforementioned residual impairment model, but they only considered hardware impairments at the transmitter side and mainly derived high-SNR capacity ceilings. Very recently, reported how hardware impairments affect dual-hop relaying systems. However, to the best of our knowledge, a detailed study of the MIMO system capacity in the presence of residual transceiver hardware impairments is missing from the literature. Motivated by the above discussion, we hereafter analytically assess the impact of residual RF impairments in the transmitter and receiver hardware of MIMO systems. More specifically, we derive a new analytical expression for the MIMO ergodic capacity in independent and identically distributed (i.i.d.) Rayleigh fading channels for arbitrary SNR values.

Additionally, we also present asymptotic capacity expressions in the high-SNR regime. In the low-SNR regime, we derive expressions for the minimum normalized energy per information bit required to convey any positive rate reliably and the wideband slope [13], which are the two key lowSNR parameters. Throughout our analysis, we find that the impact of residual impairments is marginal on low SNR systems, while it can substantially affect the performance of high SNR systems. In the last part, we analyze the ergodic capacity of large-dimensional MIMO systems with transceiver impairments and deduce asymptotic closed-form expressions for three typical cases. This provides valuable insights on how transceiver impairments affect large-scale (or "massive") MIMO systems.

Notation: Upper and lower case boldface letters denote matrices and vectors, respectively. The trace of a matrix is expressed by $\operatorname{tr}(\cdot)$. The $n \times n$ identity matrix is represented by $I_{n}$. The expectation operation is $E[\cdot]$, while the matrix determinant is $\operatorname{denoted} b y \operatorname{det}(\cdot)$. The superscripts $(\cdot) \mathrm{H}$ and $(\cdot)-1$ stand for Hermitian transpose and matrix inverse, respectively. The Euclidean vector norm is denoted by $\|$.$\| . The symbol \mathrm{CN}$ $(\mathrm{m}, \Sigma)$ denotes a circularly-symmetric complex Gaussian distribution with mean $\mathrm{m}$ and covariance $\Sigma$.

In real-world systems, signals are affected by non-idealities and imperfections. In the case of the test bed, the RF-chain in the transmit and receive-path is especially vulnerable to impairments. In this chapter, the main impairments in the transmit-path of the test bed are identified and measured. The EVM is used to determine the overall performance of the transmit chain. Then, the system model introduced is changed such that impairments in the transmit-path are also considered. The impact of impairments in the transmit-path on different MIMO detection algorithms is illustrated by simulations and measurements carried out on the offlinetest bed. And last but not least, performance measurements of the real-time test bed are shown.

\section{Outputs}

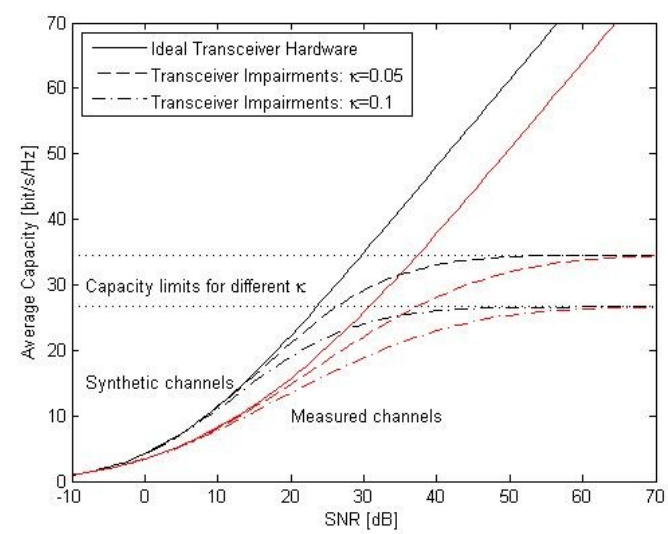

Fig 8 Avg. Capacity Vs SNR 


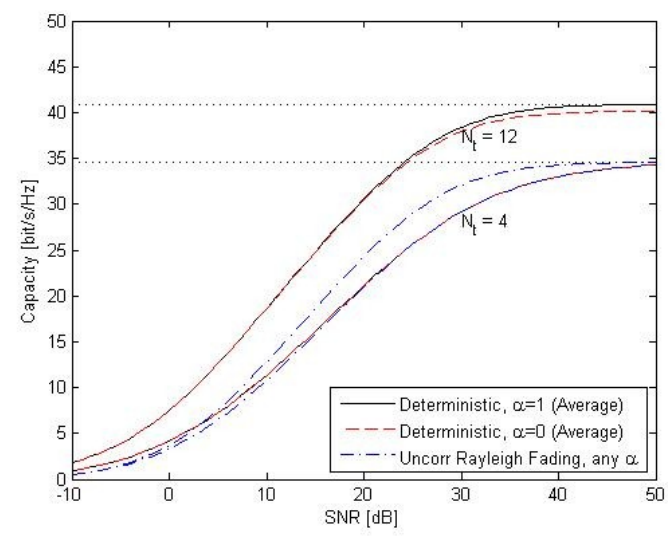

Fig 9 Capacity Vs SNR

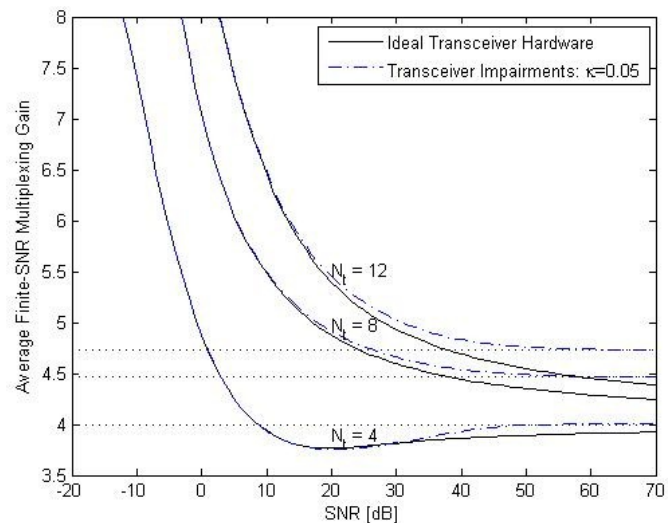

Fig 10 Avg. Finite SNR Multiplexing Vs SNR

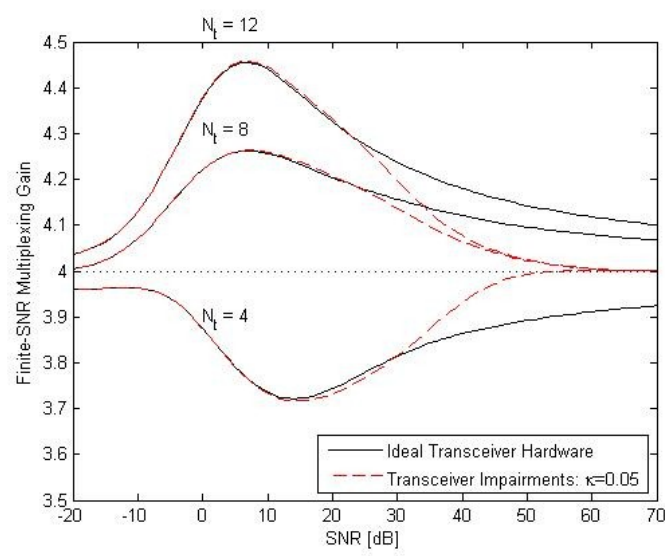

Fig 11 Finite SNR Multiplexing Vs SNR

\section{Conclusion}

Due to the lack of a fundamental metric of the performance, previous research on multiple antennas channels, especially the design of the coding schemes, is split into two different branches, focusing either on extracting the maximal diversity gain or the maximum spatial multiplexing gain. The purpose of this dissertation is to provide a unified view of the problem, by drawing a picture that connects these two types of gains. The main contribution of this dissertation is summarized as follows. We propose the new point-of-view that given a multiple antenna system both the diversity gain and the multiplexing gain can be simultaneously achieved, but there is a fundamental tradeoff between how much of each type of gain any coding scheme can achieve.

We give a concrete definition of the diversity gain and the spatial multiplexing gain for the multiple antenna channels, and a complete concept of the asymptotic analysis at high SNR. For the coherent channel model, we give a simple closed form solution of the optimal tradeoff curve, as well as a geometric interpretation that helps to understand the typical way to make detection errors in such a channel. The ability of ideal MIMO channels has a high-SNR slope that equals the minimum of the number of transceiver antennas. 
This work evaluates if this result holds when there are distortions from physical transceiver limitations. We prove analytically that such physical MIMO channels have a finite upper capacity limit, for any channel distribution and SNR. The high-SNR slope thus collapses to zero. This appears discouraging, but we prove the encouraging result that the relative capacity gain of employing MIMO is at least as large as with ideal transceivers. The entire results will be shown in MATLAB platform effectively.

The approach provides valuable insights to the resource in MIMO systems and understanding of the traditional techniques such as the union bound. We propose to use the tradeoff performance as a comprehensive performance metric. We use this metric to analysis several well-known schemes, to understand their limit and propose improvement.

\section{References}

[1] J. Winters, "On the capacity of radio communication systems with diversity in a rayleigh fading environment," IEEE JSAC, vol. 5, pp. 871-878, June 1987.

[2] B. Hassibi and B.M. Hochwald, "High-rate codes that are linear in space and time," IEEE Trans. on Inf. Theory, vol. 48, no. 7, pp. 1804-1824, July 2002.

[3] W. Yu, W. Rhee, S. Boyd, and J. Cioffi, "Iterative water-filling for vector multiple access channels," in Proc. IEEE Int. Symp. Inf. Theory, (ISIT), June 2001.

[4] C. Windpassinger, R. F. H. Fischer, and J. B. Huber, "Lattice-reduction-aided broadcast precoding," in Proc. 5th International ITG Conference on Source and Channel Coding (SCC), Erlangen, Germany, January 2004, pp. 403-408.

[5] M. Tomlinson, "New automatic equaliser employing modulo arithmetic," Electronics Letters, vol. 7, no. 5/6, pp. 138-139, March 1971.

[6] H. Harashima and H. Miyakawa, "Matched-transmission technique for channels with intersymbol interference," IEEE Trans on Comn., vol. 20, no. 4, pp. 774-780, August 1972

[7] R. Bogdan, C. Fernando, and P. T. Balsara, "Event-driven simulation and modeling of phase noise of an RF oscillator,"IEEE Trans. Circuits Syst., vol. 52, pp. 723-733, 2005.

[8] H. Bölcskei, "Blind estimation of symbol timing and carrier frequency offset in wireless OFDM systems,"'IEEE Transactions on Communications, vol. 49, pp. 988-999, 2001.

[9] "Urban transmission loss models for mobile radio in the 900-and $1800 \mathrm{MHz}$ bands," COST 231, Tech. Rep., Sept. 1991. 